\title{
Neonatal Volkmann Ischemic Contracture: Case Report and Review of Literature
}

\author{
Hitesh Agrawal, MD ${ }^{1}$ Gunjan Dokania, MD ${ }^{1}$ Shou-Yien Wu, MD²,3 \\ ${ }^{1}$ Department of Pediatrics, John H Stroger Jr. Hospital of Cook County, \\ Chicago, Illinois \\ 2 Division of Neonatology, John H Stroger Jr. Hospital of Cook County, \\ Chicago, Illinois \\ ${ }^{3}$ Department of Neonatology, Chicago Medical School, Rosalind \\ Franklin University of Medicine and Science, Chicago, Illinois \\ Address for correspondence Hitesh Agrawal, MD, Department of \\ Pediatrics, John H Stroger Jr. Hospital of Cook County, 1900 W Polk Street, \\ Room 1134, Chicago, IL 60612 (e-mail: hiteshdos@hotmail.com). \\ Am J Perinatol Rep 2014;4:e77-e80.
}

\begin{abstract}
Keywords

- neonatal Volkmann ischemic contracture

- neonatal extremity gangrene

- skin necrosis

- contracture

Background Neonatal Volkmann ischemic contracture in newborns is a devastating condition with lifelong consequences.

Case Report We report a neonate born with necrotic skin lesions and bullae on right dorsal thenar aspect of hand, who subsequently developed compartment syndrome requiring fasciotomy.

Review and Conclusion Necrotic skin lesions with/without swelling, bullae are invariably present at birth in these patients and should be recognized as a sentinel finding of underlying tissue ischemia/compartment syndrome. Early recognition and prompt surgical intervention can be limb saving. A range of radiologic abnormalities and contractures were noted in upto $84 \%$ of such patients followed long term. Hence, we recommend close follow-up until occurrence of epiphyseal fusion in these patients.
\end{abstract}

In 1881, Richard Von Volkmann described a 16-year-old patient with a deformity of hand and wrist that he ascribed to interference with forearm muscular blood flow. ${ }^{1}$ Increased compartmental pressure ensues from muscular ischemia and sets up a vicious cycle of increased pressure, nerve damage, tissue necrosis, and fibrosis. The clinical picture varies with the severity of nerve and tissue damage. The pathology is best described as a clinical spectrum ranging from transient ischemia with no major damage to compartment syndrome which if left untreated leads to Volkmann ischemic contracture depending on the degree and duration of the ischemic insult.

We report a case of neonatal Volkmann ischemic contracture/compartment syndrome and reviewed pertinent literature. Our patient fits the former part of the spectrum and this is probably from the timely surgical intervention.

\section{Case Description}

A term $3.76 \mathrm{~kg}$ male neonate was born to a 32 years old Hispanic female via emergency C-section. The mother pre- sented with decreased fetal movements for 3 to 4 days. Biophysical profile showed a score of 6 of 10 with fetal decelerations. Prenatal course was uncomplicated except for gestational diabetes-A2 controlled with diet and Glyburide. Patient was born floppy, Apgar scores were 6 and 9 at 1 and 5 minutes, respectively, and amniotic fluid was meconium stained. So, patient was intubated for meconium suctioning and persistent respiratory distress. Complete blood count showed a total white count of $44,000 / \mathrm{mm}^{3}$; hemoglobin, $14.9 \mathrm{mg} / \mathrm{dL}$; and platelets $93,000 / \mathrm{mm}^{3}$. Patient was started on ampicillin, gentamycin, and cefotaxime and continued for 5 days. Over the next 24 hours, the respiratory status improved, and patient was transitioned to nasal cannula, before weaning to room air.

Of note, a $3 \times 3.5 \mathrm{~cm}$ blister was present on the right dorsal thenar hand at birth which spontaneously burst revealing a necrotic plaque underneath. Two smaller $0.5 \times 0.5 \mathrm{~cm}$-sized necrotic lesions were noted overlying the third and fourth metacarpophalangeal joints ( - Fig. 1). The entire right upper extremity appeared swollen, erythematous with sluggish received

March 29, 2014

accepted after revision

April 29, 2014

published online

June 24, 2014
DOI http://dx.doi.org/

10.1055/s-0034-1382257. ISSN 2157-6998.
Copyright (c) 2014 by Thieme Medical Publishers, Inc., 333 Seventh Avenue, New York, NY 10001, USA. Tel: +1(212) 584-4662.
License terms

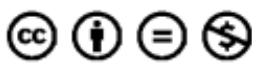




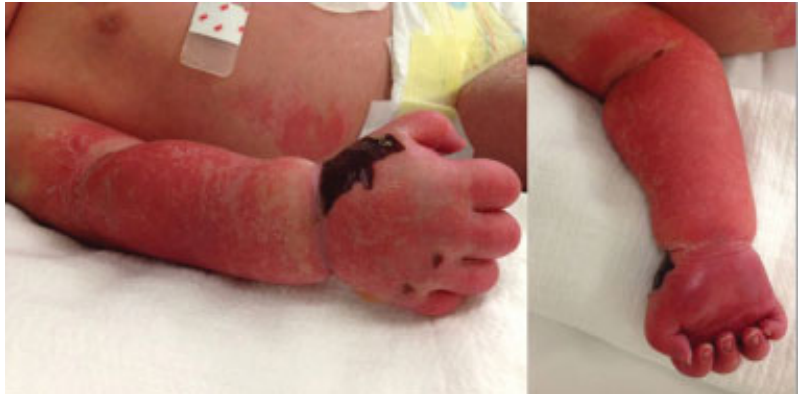

Fig. 1 Necrotic plaque on the right dorsal thenar hand with upper extremity swelling and induration at 1 day of life.

perfusion (capillary refill $10+$ seconds). The right radial pulse was absent and flaccid paralysis of right upper extremity was noted. Initial coagulation profile at 24 hours was within normal limits (prothrombin time (PT), 21.5; partial thromboplastin time (PTT), 31.4; international normalized ratio (INR), 1.96; and Fibrinogen, 208). So, patient was started on enoxaparin dosage $1.5 \mathrm{mg} / \mathrm{kg} /$ dose every 12 hours subcutaneously at 26 hours of age. For this patient only one dose of $5.7 \mathrm{mg}$ was given subcutaneously. Thereafter, progressive redness and swelling of right upper extremity was noted. Right axillary arterial thrombosis was suspected on the basis of $8 \mathrm{MHz}$ handheld Doppler. Heparin drip was started at 36 hours of age which mildly improved the perfusion. At approximately 60 hours, confirmatory doppler study of arteries and veins demonstrated normal flow proximal and distal to the lesion and no evidence of vascular thrombosis was noted. Subsequent tests were consistent with disseminated intravascular coagulation (prolonged PT/PTT, elevated INR, low fibrinogen, elevated D-dimer, low platelets, low protein C, protein S, antithrombin III, and factors II, V, VII levels). The heparin drip was stopped and patient received multiple transfusions of fresh frozen plasma and platelets before the coagulopathy resolved.

On fifth day ( $\sim 90$ hours of age), compartment pressures measured to be $20 \mathrm{~mm} \mathrm{Hg}$ on the affected arm and $15 \mathrm{~mm} \mathrm{Hg}$ on the unaffected side with persistently decreased perfusion of the right extremity. So, right forearm fasciotomy was performed next day ( $\sim 112$ hours of age) which revealed necrotic subcutaneous tissue over dorsoradial forearm. The dorsal, volar, and mobile wad compartments were released and showed pink viable muscles. A week later, the affected extremity appeared red and hard because of fat tissue necrosis requiring debridement and negative pressure wound therapy. Delayed primary closure of the skin was performed. The negative pressure wound therapy was used for 1 month with serial changes leading to clean and healthy wounds without signs of infection.

At 3 weeks of age, right upper arm radiographs demonstrated subtle lucency and irregularity along the distal metaphyses of radius and ulna (-Fig. 2). Nerve conduction studies showed no response of the right ulnar nerve, $20 \%$ response in right musculocutaneous nerve and only motor response was demonstrable in the right median nerve. Skin biopsy taken at the time of fasciotomy was negative for Kaposiform hengioendothelioma and Kasabach-Merritt phenomenon and blood cultures did not grow any organisms.

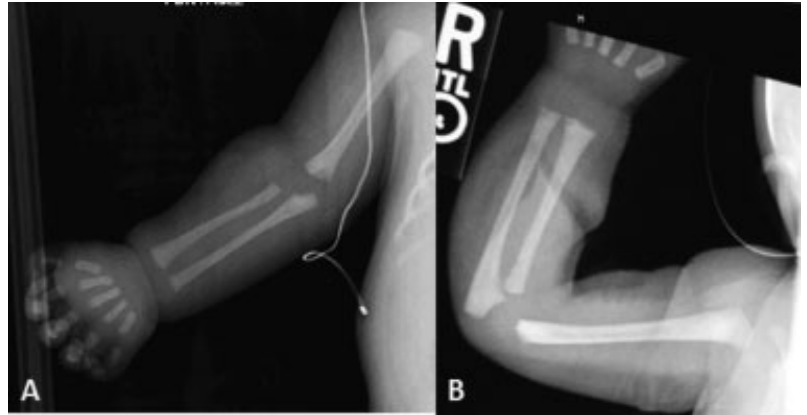

Fig. 2 (A) Right upper extremity radiograph at 10 hours of age showing normal radius and ulna with soft tissue swelling around the elbow; (B) Radiograph at 3 weeks of age demonstrating subtle lucency and irregularity along the distal metaphyses of radius and ulna.

The patient was closely followed by occupational therapist and also referred into the early intervention program. At 3 months follow-up, the affected extremity has a normal tone, good grip, and a healthy appearance (-Fig. 3), although spontaneous movements are slightly less compared with the other side.

\section{Discussion}

We found 50 cases (30 males, 17 females, and 3 unspecified) of neonatal Volkmann ischemic contracture described in the literature $^{2-20}$ (- Table 1). All occurred in the upper extremities except two cases where lower extremity was involved. ${ }^{6,20}$ In all of these cases, skin lesions were present at birth, indicating that the process started in utero. Although we could not come up with a definitive etiological agent, the clinical manifestations of all the cases fell into the same spectrum, suggesting that the pathophysiological basis may be the same.

Skin blisters on the hand evolving into necrotic plaques as seen in our case is a sentinel finding of underlying tissue

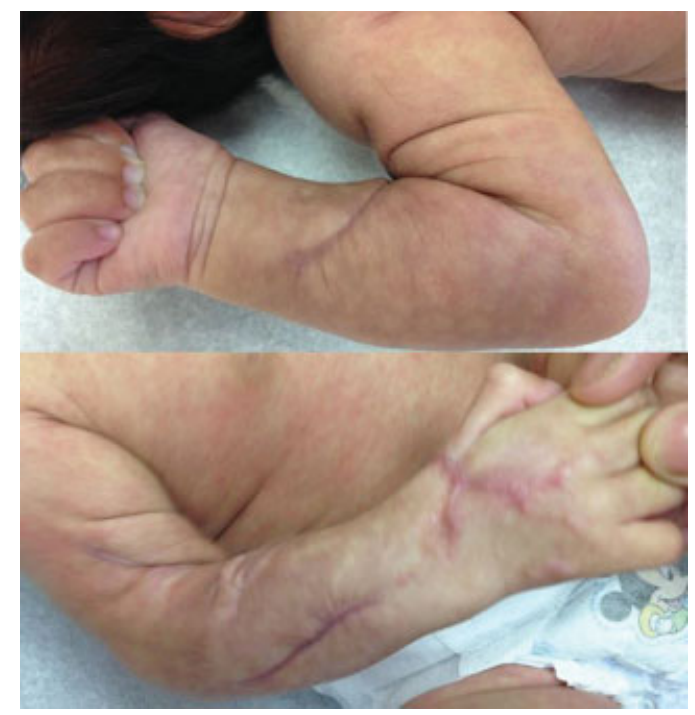

Fig. 3 Healthy appearance of right upper extremity at 3 months of life. 
Table 1 Review of previous cases of neonatal Volkmann ischemic contracture/compartment syndrome

\begin{tabular}{|c|c|}
\hline Cases & No. of patients \\
\hline \multicolumn{2}{|l|}{ Prenatal/neonatal comorbidity } \\
\hline Preterm & 9 \\
\hline Maternal DM & 7 \\
\hline Oligohydraminos & 6 \\
\hline Difficult extraction & 5 \\
\hline Abnormal fetal presentation & 5 \\
\hline Twin pregnancy & 4 \\
\hline PROM & 3 \\
\hline Fetal microsomal & 2 \\
\hline Excessive maternal weight gain & 2 \\
\hline Neonatal sepsis & 1 \\
\hline Neonatal seizures & 2 \\
\hline Neonatal asphyxia & 2 \\
\hline Maternal varicella & 2 \\
\hline \multicolumn{2}{|l|}{ Clinical presentation at birth } \\
\hline Skin necrosis & 44 \\
\hline Escher & 4 \\
\hline Cyanosis & 1 \\
\hline Erythema, blister only & 1 \\
\hline \multicolumn{2}{|l|}{ Long-term findings } \\
\hline Contractures & $38 / 45$ \\
\hline Radiologic abnormality & $32 / 39$ \\
\hline \multicolumn{2}{|l|}{ Management } \\
\hline Fasciotomy & 7 \\
\hline Late reconstructive procedure & 20 \\
\hline Surgical debridement & 4 \\
\hline No surgery & 19 \\
\hline
\end{tabular}

Abbreviations: DM, Diabetes mellitus; PROM, preterm premature rupture of membranes.

ischemia and compartment syndrome- $88 \%$ of the reported cases had skin necrosis at birth ${ }^{2-20}$ (- Table 1). This association is most often not appreciated and emergent fasciotomy has been rarely been performed..$^{9,10,13,14}$ Ragland et al ${ }^{14}$ found that larger or circumferential lesions were associated with greater tissue loss and poorer function. Depending on the clinical scenario close observation may be prudent for minor skin lesions but an untreated compartment syndrome, can quickly evolve into a Volkmann contracture with muscle infarction and nerve damage. Distal pulses are usually present, 13 patients had palpable pulses, 2 patients had no distal pulse, and for 35 patients such information was not available.

The specific cause is unknown and it is speculated that both extrinsic and intrinsic factors may play a role. Extrinsic factors include oligohydramnios, multiple gestation, fetal macrosomia, abonormal in utero posture/positioning, difficult extractions, maternal diabetes, and excessive maternal weight gain ${ }^{2-20}$ (-Table 1). In this case, maternal diabetes, fetal macrosomia and the insult leading to decreased fetal movements were the likely contributory factors. Intrinsic factors include hypercoagulable state, leading to intravenous or intra-arterial thrombosis., ${ }^{3,22}$ However, occlusion of a major blood vessel gives rise to a different clinical syndrome known as extremity gangrene of newborn. Pulses are invariably absent and the end result is amputation of the affected extremity without prompt therapy. Successful salvage with heparin, thrombolysis, or thrombectomy has been reported previously. ${ }^{23,24}$ In cases of Volkmann ischemic contracture, external compressive effects seems more plausible because of involvement of superficial extensor muscle group, overlying skin, and the return of nerve function in previous reports. The coagulopathy in our patient was likely secondary to the compressive effects in utero and possibly precipitated by the ischemic insult. This predisposition is likely due to the fact that plasma concentrations of prothrombin, several clotting factors and antithrombin III are only approximately $50 \%$ of adult concentrations in term newborns and these are even lower in premature infants. Also, newborns have dysfunctional and reduced concentration of plasminogen, tissue plasminogen activator, and normal/increased concentrations of plasminogen activator inhibitors. ${ }^{23}$

Regardless of the inciting event, the end result is an increase in compartment pressure, leading to muscle ischemia fibrosis and contractures. Of 45 patients that were followed up long term, 38 developed contractures and 32 had radiographically detectable bony abnormalities. Hence, long-term follow-up is critical for these patients. In this case, the compartmental pressure in the affected forearm was only mildly elevated compared with the other side, but the blood flow persistently remained sluggish in the right extremity and the risk of ongoing ischemia was high. Hence, a decision for fasciotomy was made.

\section{Conclusion}

Necrotic lesions of the extremities with/without skin blisters in a neonate is a sentinel finding of underlying tissue ischemia and compartment syndrome. Early recognition of such findings and emergent fasciotomy can be limb saving during the acute phase. In the long run, physical/occupational therapy and reconstructive procedures to improve the functional ability of the limb remains the mainstay of therapy and can mitigate life-long disability. Our review also emphasizes on the range of X-ray deformities and high incidence of contractures that have been reported previously. Hence, these patients should be followed closely until epiphyseal fusion occurs in the affected extremity.

\section{References}

1 Volkmann R. Die ischaemischen muskellahmungen and kontrakturen. Zentralbl Chir 1881;8:801-803

2 Allen LM, Benacci JC, Trane RN III, Driscoll RJ. A case of neonatal compartment syndrome: importance of early diagnosis in a rare and debilitating condition. Am J Perinatol 2010;27(2):103-106

3 Armstrong AP, Page RE. Intrauterine vascular deficiency of the upper limb. J Hand Surg [Br] 1997;22(5):607-611 
4 Caouette-Laberge L, Bortoluzzi P, Egerszegi EP, Marton D. Neonatal Volkmann's ischemic contracture of the forearm: a report of five cases. Plast Reconstr Surg 1992;90(4):621-628

5 Cham PM, Drolet BA, Segura AD, Esterly NB. Congenital Volkmann ischaemic contracture: a case report and review. $\mathrm{Br} J$ Dermatol 2004;150(2):357-363

6 Christiansen SD, Desai NS, Pulito AR, Slack MR. Ischemic extremities due to compartment syndromes in a septic neonate. J Pediatr Surg 1983;18(5):641-643

7 Dahlin LB, Erichs K, Rosberg HE. Compartment syndrome in the forearms of two neonates. Scand J Plast Reconstr Surg Hand Surg 2009;43(1):58-60

8 Engel J, Heim M, Tsur H. Late complications of neonatal Volkmann's ischaemia. Hand 1982;14(2):162-163

9 Isik C, Demirhan A, Karabekmez FE, Tekelioglu UY, Altunhan H, Ozlu T. Forearm compartment syndrome owing to being stuck in the birth canal: a case report. J Pediatr Surg 2012;47(11):e37-e39

10 Kline SC, Moore JR. Neonatal compartment syndrome. J Hand Surg Am 1992;17(2):256-259

11 Léauté-Labrèze C, Depaire-Duclos F, Sarlangue J, et al. Congenital cutaneous defects as complications in surviving co-twins. Aplasia cutis congenita and neonatal volkmann ischemic contracture of the forearm. Arch Dermatol 1998;134(9):1121-1124

12 Pavlidis E, Spagnoli C, Duca M, Ormitti F, Magnani C, Pisani F. Neonatal forearm compartment syndrome: look for cerebral stroke. J Pediatr 2014;164(2):e1

13 Plancq MC, Buisson P, Deroussen F, Krim G, Collet LM, Gouron R. Successful early surgical treatment in neonatal compartment syndrome: case report. J Hand Surg Am 2013;38(6):1185-1188
14 Ragland R III, Moukoko D, Ezaki M, Carter PR, Mills J. Forearm compartment syndrome in the newborn: report of 24 cases. J Hand Surg Am 2005;30(5):997-1003

15 Raimer L, McCarthy RA, Raimer D, Colome-Grimmer M. Congenital Volkmann ischemic contracture: a case report. Pediatr Dermatol 2008;25(3):352-354

16 Rios M, Ribeiro C, Soares P, et al. Volkmann ischemic contracture in a newborn. BMJ Case Rep 2011;2011:2011

17 Silfen R, Amir A, Sirota L, Hauben DJ. Congenital Volkmann-Lesser ischemic contracture of the upper limb. Ann Plast Surg 2000; 45(3):313-317

18 Tsujino A, Hooper G. Neonatal compression ischaemia of the forearm. J Hand Surg [Br] 1997;22(5):612-614

19 Tsur H, Yaffe B, Engel Y. Impending Volkmann's contracture in a newborn. Ann Plast Surg 1980;5(4):317-320

20 Van der Kaay DC, Horsch S, Duvekot JJ. Severe neonatal complication of transverse lie after preterm premature rupture of membranes. BMJ Case Rep 2013;2013:201

21 Fee HJ, McAvoy JM, Dainko EA. Neonatal arterial occlusion. J Pediatr Surg 1977;12(5):711-713

22 Wiseman NE, Briggs JN, Bolton VS. Neonatal arterial occlusion with ischemic limb gangrene. J Pediatr Surg 1977;12(5): 707-710

23 Giacoia GP. High-dose urokinase therapy in newborn infants with major vessel thrombosis. Clin Pediatr (Phila) 1993;32(4): 231-237

24 Ricciardelli E, Morgan RF, Lin KY. In utero brachial artery thrombosis: limb salvage with postnatal urokinase infusion. Ann Plast Surg 1995;34(1):81-83 